\title{
In-situ Growth of MAX Phase Coatings on Ecological Carbon and their Terahertz Shielding Properties Investigations
}

\section{Jiaxuan Huang}

Shaanxi University of Science and Technology

\section{Hujie Wan}

University of Electronic Science and Technology of China

\section{Mian Li}

Ningbo Institute of Materials Technology and Engineering Chinese Academy of Sciences: Ningbo Institute of Industrial Technology Chinese Academy of Sciences https://orcid.org/0000-0002-3139-7456

\section{Yiming Zhang}

Ningbo Institute of Materials Technology and Engineering CAS: Ningbo Institute of Industrial

Technology Chinese Academy of Sciences

Jianfeng Zhu

Shaanxi University of Science and Technology

\section{Xuelin Li}

Shaanxi University of Science and Technology

\section{Wenchao Shui}

University of Electronic Science and Technology of China

\section{Yao Li}

University of Electronic Science and Technology of China

\section{Xiaomeng Fan}

Northwestern Polytechnical University

\section{Qiye Wen}

University of Electronic Science and Technology of China

\section{Xu Xiao}

University of Electronic Science and Technology of China

Qing Huang ( $\sim$ huangqing@nimte.ac.cn )

Shaanxi University of Science and Technology https://orcid.org/0000-0001-7083-9416

\section{Research Article}

Keywords: Electromagnetic interference (EMI), MAX@CW composite, carbonized wood (CW), shielding effectiveness 
Posted Date: April 1st, 2021

DOI: https://doi.org/10.21203/rs.3.rs-376940/v1

License: (c) (i) This work is licensed under a Creative Commons Attribution 4.0 International License. Read Full License 


\section{Abstract}

Electromagnetic interference (EMI) shielding materials have received great interests in recent years. The EMI shielding effectiveness (SE) of materials not only depends on their composition, but also is influenced by their microstructures. Among various microstructure prototypes, porous structure has special advantage of low density and high electromagnetic wave reflections ability. Herein, a MAX@CW composite ( $\left.\mathrm{Ti}_{2} \mathrm{AIC} @ C W, \mathrm{~V}_{2} \mathrm{AlC} @ C W, \mathrm{Cr}_{2} \mathrm{AIC} @ C W\right)$ with porous structure is fabricated through a molten salt method by using carbonized wood (CW) as template. The MAX@CW forms conductive network constructions and rich interface that can further improve the EMI shielding effectiveness. The average EMI shielding effectiveness of the three kinds of MAX@CW with the thickness of $1 \mathrm{~mm}$ are higher than 45 $\mathrm{dB}$ in the frequency range of $0.6 \sim 1.6 \mathrm{THz}$. Among them, $\mathrm{V}_{2} \mathrm{AlC} @ \mathrm{CW}$ show the best EMI shielding effectiveness with the average value of $55 \mathrm{~dB}$.

\section{Introduction}

Owing to the rapid development of terahertz $(\mathrm{THz})$ technology, especially the designation of terahertz band as the next generation (6G) communication band, THz shielding materials are urgently demanded for avoiding malfunction in electronic devices stemming from signal crosstalk and protecting people from electromagnetic pollution. Moreover, it is preferable to develop lightweight and environment-friendly $\mathrm{THz}$ shielding materials ${ }^{[1-4]}$. In general, the effectiveness of $\mathrm{THz}$ shielding materials is highly related to their microstructure ${ }^{[5,6]}$. For instance, structures with specific interface contacts (e.g. core-shell structure, sandwich-like structure and porous foam structure) could induce interfacial polarization to dissipate electromagnetic waves, and thus improve electromagnetic shielding effectiveness ${ }^{[7-11]}$. Among them, materials with porous structures show the merits of large specific surface area, lightweight and high strength to weight ratio, making them attract great attentions.

As one kind of the porous materials, the carbonized wood (CW) obtained through high-temperature carbonization of natural wood, has been widely studied due to their characteristics of light weight, multistage pore structure and anisotropy ${ }^{[12-16]}$. The natural 3D porous conductive network makes it as a promising candidate for high-effectiveness electromagnetic interference (EMI) shielding materials ${ }^{[17-20]}$. However, the single shielding mechanism of CW limits its applicable potential in EMI shielding fields. Employing the natural skeleton shape of $\mathrm{CW}$ as a template, integrated with other types of conductive materials, to obtain functional composite structural materials is treated as a feasible tactic to improve the EMI shielding effectiveness(SE) of $\mathrm{CW}^{[21]}$.

Based on above considerations, we employ CW as the template to design a wood-based conductive composite with three-dimensional porous structure as a THz shielding material. MAX phases are a family of layered ternary transition metal carbides/nitrides, which combines the good attributes of ceramics and metals, such as superior oxidation and corrosion resistance, eminent electrical/thermal conductivity, and high dielectric loss. With these virtue, MAX phases are proposed to be ideal candidates as EMI shielding 
materials for harsh environment applications ${ }^{[22-24]}$. Herein, a MAX@CW composite $\left(\mathrm{Ti}_{2} \mathrm{AIC} @ C W\right.$, $\mathrm{V}_{2} \mathrm{AIC} @ \mathrm{CW}, \mathrm{Cr}_{2} \mathrm{AIC} @ \mathrm{CW}$ ) with porous structure is fabricated through in-situ growth MAX phase coating on the CW in a molten salt bath. The MAX@CW retains the characteristics of porous structure and anisotropy from carbonized wood template. Meanwhile, the carbonized wood template guides the directional assembly of the MAX phase by making it filling into the porous structure of the CW, showing a multi-interfaces. The MAX@CW with the specific structure is supposed to manifold the electromagnetic dissipation paths of electromagnetic wave within the porous network structure, which greatly increased transmission loss and improved its EMI SE. As a result, the MAX@CW should much enhanced SE than the CW and show great potential as a high-effectiveness EMI shielding material.

\section{Experimental Procedures \\ 2.1 The synthesis of CW}

Firstly, the natural linden is cut into pieces and put them into an alumina crucible. And then the hightemperature carbonization is carried out at $1000^{\circ} \mathrm{C}$ for $6 \mathrm{~h}$ in argon atmosphere. After that, the carbonized wood slices are carefully polished with 1000 mesh sand-tray to obtain ones with thickness of $1 \mathrm{~mm}$. Subsequently, the residual carbon is removed by ultrasonic washing with deionized water and ethanol for many times. The final CW samples can be obtained after drying in an ordinary oven.

\subsection{Synthesis of MAX@CW}

The MAX@CW (The MAX phases in use include $\mathrm{Ti}_{2} \mathrm{AIC}, \mathrm{V}_{2} \mathrm{AIC}, \mathrm{Cr}_{2} \mathrm{AIC}$ ) are prepared by molten salt method. The $\mathrm{CW}$ is used both as template and carbon source. The $\mathrm{M}$-site metal powder (i.e. $\mathrm{Ti}, \mathrm{V}$ and $\mathrm{Cr}$ ) and A-site metal powder (Al) with both of their particle size of $\sim 1 \mu \mathrm{m}$ are used as the raw materials to synthesize the target MAX@CW. In addition, the analytical grade $\mathrm{NaCl} / \mathrm{KCl}$ are selected for the molten salt bath, purchased from Shanghai Aladdin Industrial Co., Ltd.

The in-situ preparation of MAX@CW is carried out on porous carbonized wood matrix within the salt bath. The steps are as follow: firstly, the raw material powders are mixed evenly, aiming to cover the $\mathrm{CW}$ completely; then, the MAX@CW is prepared from high-temperature sintering in argon atmosphere, where the sintering temperature for $\mathrm{Ti}_{2} \mathrm{AlC} @ C W$ and $\mathrm{V}_{2} \mathrm{AlC} @ \mathrm{CW}$ are $1000{ }^{\circ} \mathrm{C}$ (holding for $6 \mathrm{~h}$ ), and of $\mathrm{Cr}_{2} \mathrm{AlC} @ \mathrm{CW}$ is $900{ }^{\circ} \mathrm{C}$; after that, the product is washed for many times with deionized water to remove the salt under the room temperature. The final MAX@CW is obtained after drying.

\subsection{Characterization}

The derived sample is analyzed by Bruker D8 Discovery X-ray diffractometer (XRD, irradiated by Cu Ka ( $\lambda$ $=1.5406 \AA$ ) , accelerated voltage $40 \mathrm{kV}$, filament current $40 \mathrm{~mA}$, and the $2 \theta$ range of $10 \sim 70^{\circ}$ again with a step size of $0.02^{\circ}$. The structure and vibration characteristics of CW and MAX@CW are analyzed by confocal Raman microscope (Renishaw Invia Reflex) with the excitation wavelength of $532 \mathrm{~nm}$. A scanning electron microscope (SEM, Quanta FEG 250, FEI) is performed to characterize the morphology 
and microstructure of the CW and MAX@CW. The electrical conductivity of the material is measured by a four-probe detector (cresbox, Napson). The Terahertz time-domain spectroscopy (THZ-TDS) test is carried out under the conditions of room temperature $22{ }^{\circ} \mathrm{C}$ and humidity of $5 \%$ (French i2S group company, model of TZCAM). The total spectral range is $0.2-3.0 \mathrm{THz}$ (effective spectrum range: $0.3-1.65 \mathrm{THz}$ ); the repetition frequency is $1 \mathrm{kHz}$; and the size of the sample tested is $2 \times 3 \mathrm{~cm}$ with thickness of $1 \mathrm{~mm}$.

\section{Results And Discussion}

\subsection{Materials Characterization}

The composition of CW and MAX@CW is confirmed by XRD and Raman spectra. As shown in Fig. 1A, the $\mathrm{CW}$ has two wide diffraction peaks at about $23^{\circ}$ and $44^{\circ}$, which means that the $\mathrm{CW}$ obtained is amorphous ${ }^{[25]}$. For the MAX@CW samples, the peaks of CW disappear and the diffraction peaks of $\mathrm{Ti}_{2} \mathrm{AlC}, \mathrm{V}_{2} \mathrm{AlC}$ and $\mathrm{Cr}_{2} \mathrm{AlC}$ appear, indicating the formation of corresponding MAX phase coatings. The Raman spectra are more sensitive to the surface change of materials and thus are employed to detect the formation of CW and MAX@CW. As shown in Fig. 2B, the CW has two strong characteristic peaks near $1350 \mathrm{~cm}^{-1}$ and $1600 \mathrm{~cm}^{-1}$, which represent the $\mathrm{D}$ peak and $\mathrm{G}$ peak of carbon ${ }^{[26]}$. For the $\mathrm{Ti}_{2} \mathrm{AlC} @ \mathrm{CW}$ sample, the peaks at 260-270 and $360 \mathrm{~cm}^{-1}$ correspond to the $\omega_{2}, \omega_{3}$ and $\omega_{4}$ vibration mode of $\mathrm{Ti}_{2} \mathrm{AIC}$ is observed, indicating the formation of $\mathrm{Ti}_{2} \mathrm{AlC}$. For the $\mathrm{V}_{2} \mathrm{AIC} @ \mathrm{CW}$ and $\mathrm{Cr}_{2} \mathrm{AIC} @ \mathrm{CW}$ samples, the characteristic peaks of $\mathrm{V}_{2} \mathrm{AIC}$ and $\mathrm{Cr}_{2} \mathrm{AIC}$ were also detected, which confirms their composition ${ }^{[27,28]}$.

The schematic of the fabrication process of MAX@CW composites is shown in Fig. 2A. The synthesis route consists of two steps. The first step is preparing $\mathrm{CW}$ with porous structure by carbonizing natural linden wood at high temperature. The following step is in-situ growing of MAX phase coatings on $\mathrm{CW}$ template by high-temperature molten salt reaction. The metallic elements existing as ionic form in the molten salt media can infiltrate into the pores of carbonized wood and react with carbon matrix to form MAX phase, which is driven by the pressure difference between inside and outside of the pores of carbonized wood (i.e. the capillary effect) ${ }^{[29,30]}$.

The optical photo of the product is shown in Fig. 2B, we can see that the color of CW is black, while MAX@CW has metallic luster, indicating that MAX phase coating is successfully prepared on the carbonized wood substrate. As shown in Fig. $2 \mathrm{C}-\mathrm{F}$, the $\mathrm{CW}$ has a rich pore structure, this is because that linden is a kind of natural organic polymer compounds, mainly composed of cellulose, hemicellulose and lignin. These substances will further decomposed and evaporated during the process of high temperature carbonization, leaving honeycomb pore structures of different length-scales from nanometer to micron. The pore shapes of $\mathrm{CW}$ are mainly oval with large pores of $30 \sim 60 \mu \mathrm{m}$ and small pores of $10 \sim 15 \mu \mathrm{m}$. In addition, as shown in Fig. 2E-F, the channels of $\mathrm{CW}$ are long and straight, which is favorable for the impregnation and infiltration of MAX phase coating. Since the microstructure of $\mathrm{Ti}_{2} \mathrm{AIC} @ \mathrm{CW}, \mathrm{V}_{2} \mathrm{AlC} @ \mathrm{CW}$ and $\mathrm{Cr}_{2} \mathrm{AlC} @ \mathrm{CW}$ is similar, we choose $\mathrm{Ti}_{2} \mathrm{AlC} @ \mathrm{CW}$ as the representative to show their microstructure. As shown in Fig. 2G-J, owing to the atomic-level reaction in the molten salt, $\mathrm{Ti}_{2} \mathrm{AIC} @ \mathrm{CW}$ inherits the natural 
porous structure of CW, without morphological distortion and pore blockage. In addition, through Fig. 2G$\mathrm{H}$, we can see that the surface of MAX@CW samples is different from that of smooth carbonized wood substrate.The MAX@CW with this porous structure has a large number of surfaces and interfaces, which can increase the transmission path of incident electromagnetic waves and result in multiple scattering and increasing the attenuation of electromagnetic waves.

\subsection{EMI shielding performance}

The electromagnetic interference shielding effectiveness (EMI SE) of CW and three kinds of MAX@CW ( $\mathrm{Ti}_{2} \mathrm{AlC} @ \mathrm{CW}, \mathrm{Cr}_{2} \mathrm{AlC} @ \mathrm{CW}, \mathrm{V}_{2} \mathrm{AlC} @ \mathrm{CW}$ ) are shown in Fig. 3. With the thickness of $1 \mathrm{~mm}$, the average EMI SE in the frequency range of $0.6 \sim 1.6 \mathrm{THz}$ of all the three kinds of MAX@CW is higher than $45 \mathrm{~dB}$, which have been improved comparing to the CW ( $42 \mathrm{~dB})$. Among them, $\mathrm{V}_{2} \mathrm{AlC} @ \mathrm{CW}$ show the highest EMI shielding effectiveness with the average value of $\sim 55 \mathrm{~dB}$. Notably, as shown in Fig. 3B-3C, the dominant shielding mechanism of $\mathrm{CW}$ is EMW absorption with the average absorption coefficient of $79 \%$, while that value of $\mathrm{Ti}_{2} \mathrm{AlC} @ \mathrm{CW}, \mathrm{Cr}_{2} \mathrm{AlC} @ \mathrm{CW}, \mathrm{V}_{2} \mathrm{AlC} @ \mathrm{CW}$ is 36\%, 42\%, and 23\%. On the contrary, $\mathrm{Ti}_{2} \mathrm{AlC} @ \mathrm{CW}$, $\mathrm{Cr}_{2}$ AlC@CW, $\mathrm{V}_{2}$ AIC@CW show high reflection coefficient of 64\%,58\%, and 77\%.

The shielding mechanism change is caused by to the structure evolution from CW to MAX@CW. For the $\mathrm{CW}$, the highly porous structure allows electromagnetic waves to easily enter into the low reflection wood channel and be attenuated multi-reflection inner the structure ${ }^{[18]}$. While when the MAX phase coating is fabricated on the $\mathrm{CW}$ channel, the pore size is reduced that decreased the ratio of incident electromagnetic waves. Another important reason is the impedance matching property is changed after the MAX phases coating fabrication. Owing to the porous structure and relatively low dielectric constant, CW has better impedance matching property with the free space than the MAX@CW, which allows more electromagnetic waves entering into the channel, and enhanced the ration of higher EMW absorption. In contrast, MAX phase with high dielectric constant greatly increased the impedance mismatching between the free space and MAX@CW, which result in more incident electromagnetic waves being reflected at the surface ${ }^{[24,31]}$.

In order to further understand the EMI shielding mechanism of the MAX@CW, the electrical conductivities of the materials were measured. As shown in in Fig. 3D, $\mathrm{V}_{2} \mathrm{AlC} @ \mathrm{CW}$ shows the highest electrical conductivity among the three kinds of MAX@CW. As we know, the increased conductivity can result in higher impedance mismatch between the free space and MAX@CW interface, which can contribute to higher reflection loss and increase its EMI shielding effectiveness ${ }^{[32]}$. In addition, when the electromagnetic wave enters the body of the material, the higher conductivity will lead to a larger eddy current and converts EMW energy into Joule heat, which can improve the electromagnetic wave absorption loss ${ }^{[33]}$. Therefore, the MAX@CW show enhanced EMI shielding effectiveness than the CW, and $\mathrm{V}_{2} \mathrm{AlC} @ \mathrm{CW}$ shows the best shielding effectiveness.

\section{Conclusions}


In conclusion, MAX@CW composites were prepared on carbonized wood templates by the in-situ reaction in molten salt bath. The as-obtained MAX@CW composites inherit the specific microstructure of carbonized wood template, showing a three-dimensional interpenetrating pore structure and high available specific surface areas. The MAX@CW with porous structure forms continuous conductive network structure, which can increase the transmission paths for incident electromagnetic waves, achieving multiple reflections and increasing the attenuation of electromagnetic waves. Therefore, MAX@CW show excellent electromagnetic shielding performance in $\mathrm{THz}$ band. Within the frequency range of $0.6-1.6 \mathrm{THz}$, the total electromagnetic shielding effectiveness of the three kinds of MAX@CW are higher than $45 \mathrm{~dB}$, and the $\mathrm{V}_{2} \mathrm{AIC} @ \mathrm{CW}$ has the highest EMI SE that higher than $55 \mathrm{~dB}$. The results indicate the large potential of developing functional materials by using natural biological structure as templates.

\section{Declarations}

\section{Acknowledgements}

This study was supported financially by the National Natural Science Foundation of China (Grant Nos. 51902320, and U2004212). Qing Huang thanks International Partnership Program of Chinese Academy of Sciences (Grant No. 174433KYSB20190019), Leading Innovative and Entrepreneur Team Introduction Program of Zhejiang (Grant No. 2019R01003). Mian Li acknowledges the support from the fund of the State Key Laboratory of Solidification Processing in NPU (Grant No.SKLSP201917).

\section{References}

1. Shahzad F, Alhabed M, Hatter $C$ et al (2016) Electromagnetic interference shielding with $2 D$ transition metal carbides (MXenes). Science 353:1137-1140

2. Amin M, Siddiqui O, Abutarboush $\mathrm{H}$ et al (2021) A THz Graphene Metasurface for Polarization Selective Virus Sensing. Carbon 176:580-591

3. Cheng KB, Ramakrishna S, Lee KC (2000) et al. Electromagnetic shielding effectiveness of copper/glass fiber knitted fabric reinforced polypropylene composites. Compos Pt A-Appl Sci Manuf 31:1039-1045

4. Tian MW, Du MZ, Qu LJ et al (2017) Electromagnetic interference shielding cotton fabrics with high electrical conductivity and electrical heating behavior via layer-by-layer self-assembly route. Rsc Advances 7:42641-42652

5. Zhao HB, Cheng JB, Wang YZ (2018) Biomass-derived Co@crystalline carbon@carbon aerogel composite with enhanced thermal stability and strong microwave absorption performance. J Alloy Compd 736:71-79

6. Xia T, Zhang C, Oyler NA et al (2013) Hydrogenated TiO2 nanocrystals: a novel microwave absorbing material. Adv Mater 25:6905-6910 
7. Han MK, Yi XW, Li XL et al (2017) Laminated and two-dimensional carbon-supported microwave absorbers derived from MXenes. ACS Appl Mater Interfaces 9:20038-20045

8. Ohlan A, Singh K, Chandra A et al (2010) Microwave absorption behavior of core-shell structured poly (3,4-ethylenedioxy thiophene)-barium ferrite nanocomposites. Acs Appl Mater Interfaces 2:927-933

9. Han MK, Yin XW, Kong L et al (2014) Graphene-wrapped ZnO hollow spheres with enhanced electromagnetic wave absorption properties. J Mater Chem A 2:16403-16409

10. Zeng Q, Xu DW, Chen P et al (2018) 3D graphene-Ni microspheres with excellent microwave absorption and corrosion resistance properties. J Mater Sci-Mater Electron 29:2421-2433

11. Zhang WJ, Zhang ZD, Jiang YL et al (2020) Porous Fe@Fe $\mathrm{O}_{4}-\mathrm{C}$ nanocomposite using polyvinyl alcohol sponge as template for microwave absorption. J Electron Mater 49:6394-6402

12. Song JW, Chen CJ, Zhu SZ et al (2018) Processing bulk natural wood into a high-performance structural material. Nature 554:224-228

13. Chen FJ, Gong AS, Zhu MW et al (2017) Mesoporous, three-dimensional wood membrane decorated with nanoparticles for highly efficient water treatment. ACS Nano 11:4275-4282

14. Kurosaki F, Ishimaru K, Hata T et al (2003) Microstructure of wood charcoal prepared by flash heating. Carbon 41:3057-3062

15. Shibata K, Okabe T, Saito K et al (1997) Electromagnetic shielding properties of woodceramics made from wastepaper. J Porous Mat 4:269-275

16. Vartapetyan RS, Voloshchuk AM, Buryak AK et al (2005) Water vapor adsorption on chars and active carbons-oxygen sensors prepared from a tropical tree wood. Carbon 43:2152-2159

17. Hemanth J (2009) Quartz $\left(\mathrm{SiO}_{2 \mathrm{P}}\right)$ reinforced chilled metal matrix composite (CMMC) for automotive applications. Mater Des 30:323-329

18. Xi JB, Zhou EZ, Liu YJ et al (2017) Wood-based straightway channel structure for high performance microwave absorption. Carbon 124:492-498

19. Wang SY, Hung CP (2003) Electromagnetic shielding efficiency of the electric field of charcoal from six wood species. J Wood Sci 49:450-454

20. Wang LL, Tay BK, See KY et al (2009) Electromagnetic interference shielding effectiveness of carbonbased materials prepared by screen printing. Carbon 47:1905-1910

21. Du X, Zhang Z, Liu W et al (2017) Nanocellulose-based conductive materials and their emerging applications in energy devices - A review. Nano Energy 35:299-320

22. Barsoum MW (2000) The $M_{N+1} A X_{N}$ phases: A new class of solids. Prog Solid State Chem 28:201281

23. Barsoum MW, El-Raghy T (2001) The MAX phases: unique new carbide and nitride materials. Am Scientist 89:334-343

24. Mu Y, Zhou WC, Wan F et al (2015) High-temperature dielectric and electromagnetic interference shielding properties of $\mathrm{SiC}_{\mathrm{f}} / \mathrm{SiC}$ composites using $\mathrm{Ti}_{3} \mathrm{SiC}_{2}$ as inert filler. Compos Pt A-Appl Sci Manuf 77:195-203 
25. Onodera A, Terashima K, Urushihara T et al (1997) High-pressure synthesis of diamond from phenolic resin[J]. J Mater Sci 32:4309-4318

26. Robertson JR (2002) Diamond-like amorphous carbon. Mater Sci Eng R Rep 37:129-281

27. Volker $P$, Michael $N$, Laurent $C$ et al (2012) First-order Raman scattering of the MAX phases: $\mathrm{Ti}_{2} \mathrm{AIN}$, $\mathrm{Ti}_{2} \mathrm{AlC}_{0.5} \mathrm{~N}_{0.5}, \mathrm{Ti}_{2} \mathrm{AlC},\left(\mathrm{Ti}_{0.5} \mathrm{~V}_{0.5}\right){ }_{2} \mathrm{AlC}, \mathrm{V}_{2} \mathrm{AlC} \mathrm{Ti}_{3} \mathrm{AlC}_{2}$, and $\mathrm{Ti}_{3} \mathrm{GeC}_{2}$. J Raman Spectrosc 43:168-172

28. Wang JY, Zhou YC, Lin ZJ et al (2005) Raman active phonon modes and heat capacities of $\mathrm{Ti}_{2} \mathrm{AIC}$ and $\mathrm{Cr}_{2} \mathrm{AlC}$ ceramics: first-principles and experimental investigations. Appl Phys Lett 86:201-204

29. Stamm AJ (1931) Three methods of studying capillary structure as applied to wood. Physics 1:116128

30. Kang W, Chung WY (2009) Liquid water diffusivity of wood from the capillary pressure-moisture relation. J Wood Sci 55:91-99

31. Shui W, Li J, Wang H et al (2020) $\mathrm{Ti}_{3} \mathrm{C}_{2} \mathrm{~T}_{x}$ MXene sponge composite as broadband terahertz absorber. Adv Opt Mater 8:20011-20020

32. Zeng S, Li X, Li M et al (2019) Flexible PVDF/CNTs/Ni@CNTs composite films possessing excellent electromagnetic interference shielding and mechanical properties under heat treatment. Carbon 155:34-43

33. Zhao B, Wang S, Zhao C et al (2018) Synergism between carbon materials and Ni chains in flexible poly (vinylidene fluoride) composite films with high heat dissipation to improve electromagnetic shielding properties. Carbon 127:469-478

\section{Figures}
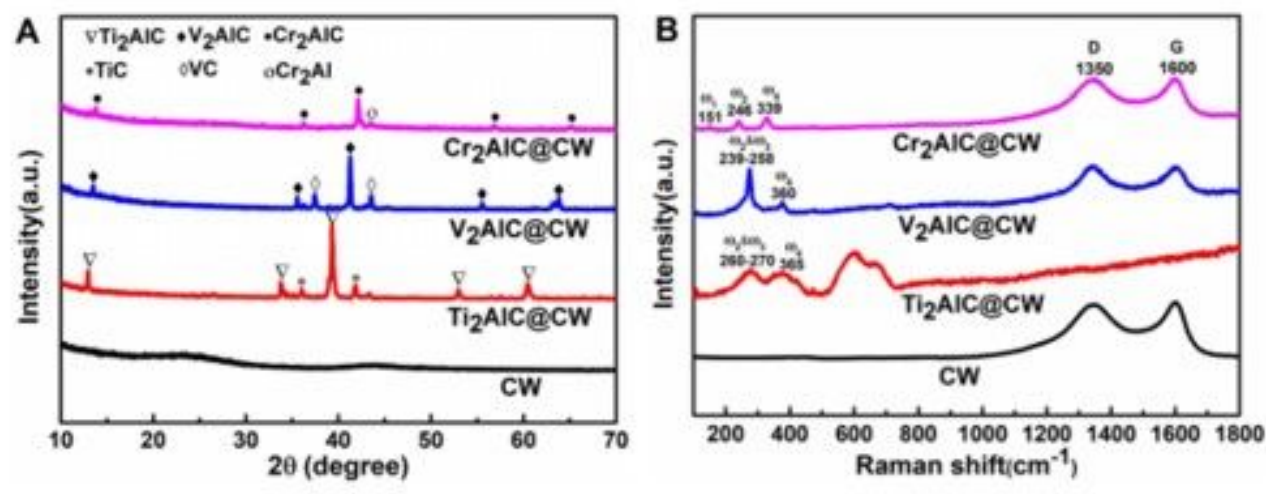

Figure 1

XRD patterns (A) and Raman spectra (B) of CW and MAX@CW 


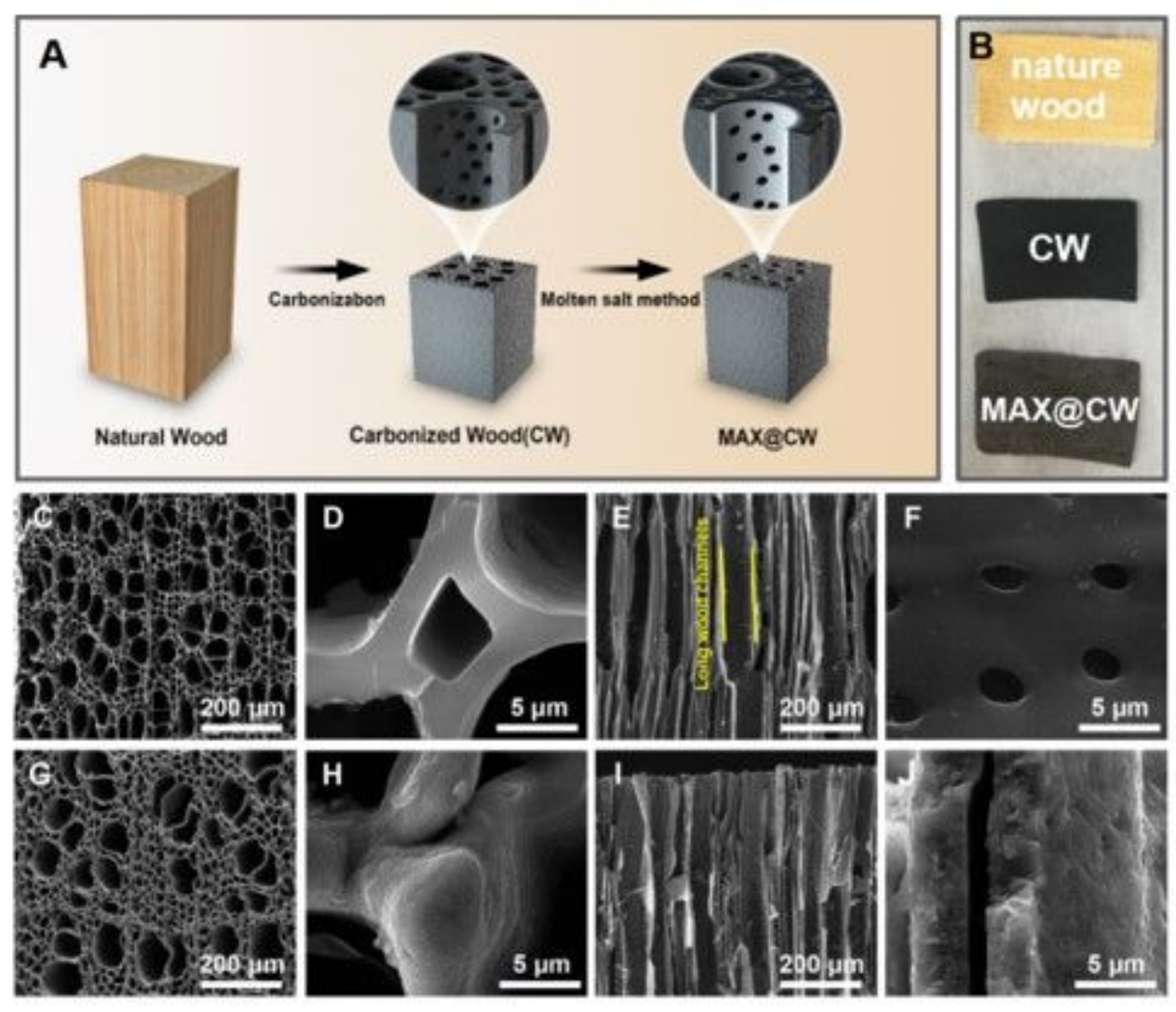

Figure 2

Schematic illustration of the fabrication process and macro- and microstructure of sample. (A) Schematic illustration of the fabrication process, (B) Optical photos of nature, CW and MAX@CW, (C-F) SEM image of bare CW, (G-J) SEM image of Ti2AIC@CW. 

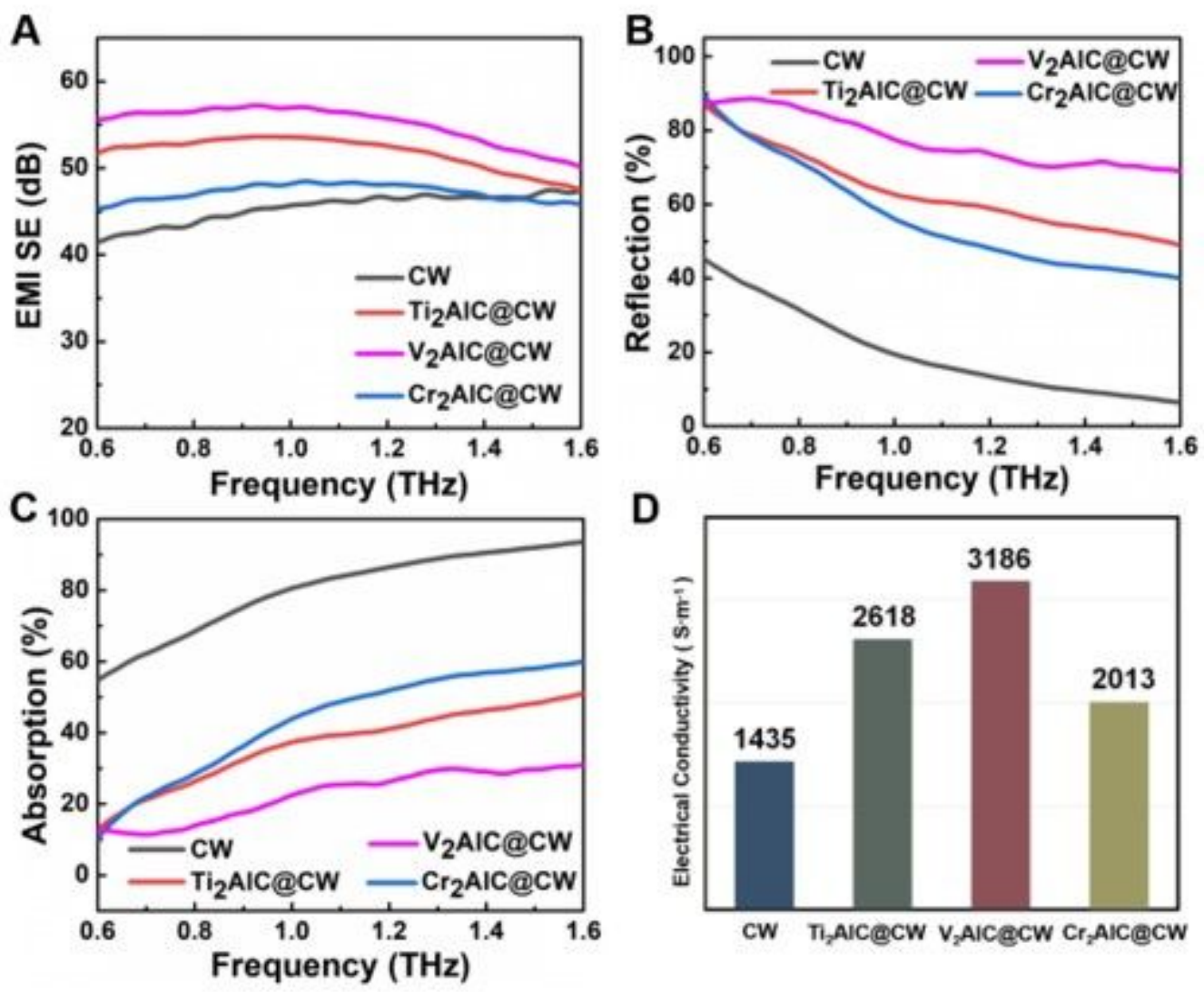

Figure 3

The EMI SE and conductivity of CW and MAX@CW with thickness of $1 \mathrm{~mm}$ in perpendicular channel direction. (A) Total EMI SE of CW and MAX@CW, (B) Reflection of CW and MAX@CW, (C) Absorption of CW and MAX@CW, (D) Conductivity of CW and MAX@CW. 